\title{
Human Fall Detection Using Machine Learning Methods: A Survey
}

\author{
Komal Singh \\ Department of Computer Science and Engineering, \\ Graphic Era Deemed to be University, Dehradun, Uttarakhand, India. \\ Corresponding author: komalsingh.0492@gmail.com \\ Akshay Rajput \\ Department of Computer Science and Engineering, \\ Graphic Era Deemed to be University, Dehradun, Uttarakhand, India. \\ E-mail: akshay.rajput.1711@gmail.com \\ Sachin Sharma \\ Department of Computer Science and Engineering, \\ Graphic Era Deemed to be University, Dehradun, Uttarakhand, India. \\ E-mail: sxsharma88@gmail.com
}

(Received October 11, 2018; Accepted August 12, 2019)

\begin{abstract}
Human fall due to an accident can cause heavy injuries which may lead to a major medical issue for elderly people. With the introduction of new advanced technologies in the healthcare sector, an alarm system can be developed to detect a human fall. This paper summarizes various human fall detection methods and techniques, through observing people's daily routine activities. A human fall detection system can be designed using one of these technologies: wearable based device, context-aware based and vision based methods. In this paper, we discuss different machine learning models designed to detect human fall using these techniques. These models have already been designed to discriminate fall from activities of daily living (ADL) like walking, moving, sitting, standing, lying and bending. This paper is aimed at analyzing the effectiveness of these machine learning algorithms for the detection of human fall.
\end{abstract}

Keywords- Hidden Markov model, Gaussian distribution, Multilayer perceptron, Fuzzy rule, Deep learning.

\section{Introduction}

Human falls are considered as dreaded events since they can affect a person physically as well as psychologically. Compared to younger people, older people have a greater risk of falling (Duque, 2016). As the age of a human being advances, his body becomes physically weak which increases the risk to fall. By 2030, the worldwide population having age more than 60 is predicted to grow up to 1.4 billion (WPA, 2017) and by 2050, it is predicted to grow from 962 million to 2.1 billion, relative to the year 2017 (Uddin et al., 2018). Also, according to the report WHO (2008), approx $28 \%$ to $35 \%$ of people in the age group of more than 65 years fall at least once every year. This number increases $32 \%$ to $42 \%$ for people having age more than 70 years (WHO, 2008). The major concern here is how to handle an aging population and provide them with effective healthcare services (Singh, 2014; Frieson, 2016) since in the worst cases, the fall of older people can obstruct the independence of their life and make them dependent on other human beings for the rest of their life. One of the solutions includes the presence of a person for assistance and personal care. But physically it is not possible for any person to remain present with an elderly person every time. There may be some scenarios when elderly people are left alone by their relatives. Also, there may be some cases where elderly people are prolonged living alone in their homes. It is dangerous to 
International Journal of Mathematical, Engineering and Management Sciences

Vol. 5, No. 1, 161-180, 2020

https://doi.org/10.33889/IJMEMS.2020.5.1.014

fall when alone because rescue time may increase significantly. In order to maintain their safety and to detect whether they have fallen or not when they are left alone, automatic fall detection systems are developed.

The main motive behind designing these systems is to quickly detect and recognize the fall and notify the emergency contact person for rescue as soon as possible so that not only the rescue time minimizes but also the damage due to fall minimizes (Wang et al., 2014). One of the main advantages of these systems is that they are so intelligent that they communicate with the emergency contact immediately whenever a fall is detected even in the cases where the patient is unconscious or unable to get up after the fall (Wang et al., 2008; Sposaro and Tyson, 2009; Hijaz et al., 2010; de Miguel et al., 2017). Hence, these systems increase the independent living ability of elderly people i.e. elderly people who previously, were dependent on another human being, now don't require any manual support in terms of the presence of another person all the time.

In this paper, we summarize the work done in the field of human fall detection. Three different technologies are used to develop a human-fall detection system (Igual et al., 2013; Mubashir et al., 2013; Mohamed et al., 2014; Zhu et al., 2015): (i) wearable based device, (ii) context-aware fall detection systems and (iii) vision-based systems. Section II of this paper describes these technologies in detail along with their sub categories. Section III provides a review of machine learning based human fall detection system where machine learning classification methods are combined with the above-mentioned technologies in order to develop an intelligent human fall detection system. This section also summarizes and compares fall detection algorithms designed using deep learning in vision-based systems. Finally, Section IV concludes the paper.

\section{Classification of Fall Detection Technologies}

In order to fulfill the main objective of human fall detection systems, they need to be trained so that they can discriminate between a human fall and other activities of daily life (ADL) (walking, standing, sitting, lying) (Li et al., 2009). Discrimination between a fall and an ADL is not an easy task since certain ADLs, like lying on a floor or sitting on a floor from a standing position generate strong similarities to falls. Hence, for working accurately these systems need to be trained from the data collected from falls and an ADL. This data can be collected from different types of sensors installed in the environment such as pressure sensors, floor vibration sensors, infrared sensors, microphones, and cameras. The data collected in the form of acceleration signals, pressure signals, audio, or videos are then processed and passed to the classifier which then classifies whether the collected data represents a fall or an ADL.

Depending upon the source of data collection, human fall-detection systems are categorized into three broad categories (Nizam et al., 2016), as shown in Figure 1: (i) wearable device based system, (ii) context-aware based system (non-wearable device system) and (iii) vision based system. The performance of the systems designed under these three categories is sometimes expressed either in terms of accuracy (AC) or in terms of specificity (SP) and sensitivity (SE) or in terms of precision (PR) and recall (RE) (Li et al., 2009; Abbate et al., 2010). The specificity of a model is defined as its ability to correctly detect and classify an ADL as an ADL. While the sensitivity of a model is defined as its ability to correctly detect and classify a fall as a fall. Sensitivity is also called as recall. The accuracy of a model is its ability to correctly detect a fall and an ADL. Precision is calculated as the total number of correctly detected falls divided by the total number of falls detected. 
International Journal of Mathematical, Engineering and Management Sciences

Vol. 5, No. 1, 161-180, 2020

https://doi.org/10.33889/IJMEMS.2020.5.1.014

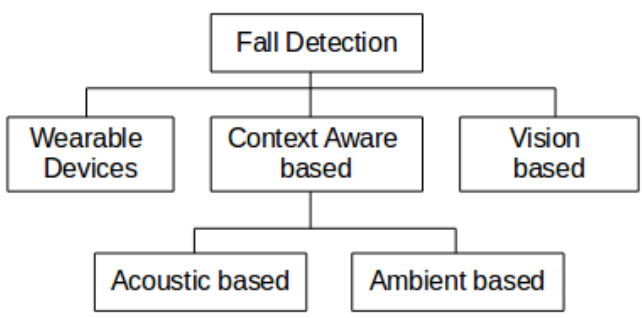

Figure 1. Classification of fall detection methods

\subsection{Wearable Device based Fall Detection Systems}

Wearable fall detection devices are electronic devices that are worn by a person under or on top of their clothes (Nizam et al., 2016). These devices comprise different types of sensors such as gyroscopes, accelerometers, tilt-meters, and oscilloscopes to detect a fall. A person has to carry the device with him all the time. Hence the elderly get location independence as they can move with the monitoring device (Doukas et al., 2007; Chen et al., 2010). But in reality, sometimes it is not possible like if the person forgets to wear the device or forgets to charge its battery or feels uncomfortable to wear it. Because of these limitations, developers direct their research towards developing contact-free fall detection systems where the user does not bother to wear anything additional for their safety (Uddin et al., 2018).

\subsection{Context-aware Fall Detection Systems}

These systems include sensors which are deployed in the environment around the humans to detect a fall. Systems designed based on this class of technology include ambience (Kim and Ling, 2009; Tzeng et al., 2010; Mastorakis and Makris, 2014) and acoustic sensors (Zhuang et al., 2009; Li et al., 2012) such as pressure sensors, floor sensors, infrared sensors, and microphones. These systems consist of a set of the above mentioned sensors to collect data. Along with them, a dedicated PC is attached. The collected data is passed to the PC for further processing and analysis (Popescu et al., 2008). The algorithm running in PC, based on certain threshold values and conditions decides whether a fall has taken place or not. Machine learning classification algorithms are also being used for the classification of human activity in two classes i.e. fall and non-fall. Systems developed under this category are further divided into two sub categories depending on the type of data collected by these sensors: (a) ambient-based fall detection systems for numerical sensor data and (b) acoustic-based fall detection systems for audio data.

\subsubsection{Ambient-based Fall Detection Systems}

This subcategory includes those systems which are designed using ambient sensors like floor sensor (Alwan et al., 2006; Zigel et al., 2009), pressure sensor (Mastorakis and Makris, 2014) and an infrared sensor (Toreyin et al., 2007). Floor and pressure sensors have to be installed on the ground so that they can read the frequency of vibrations generated during the fall. Along with them, an infrared sensor is also used to detect motion in the surrounding environment. These systems are environment dependent since every house has a different flooring. Hence, specific configurations are required for their setup which makes it difficult to install them.

\subsubsection{Acoustic-Based Fall Detection Systems}

Systems designed under this category use multiple microphones installed in the environment to 
International Journal of Mathematical, Engineering and Management Sciences

Vol. 5, No. 1, 161-180, 2020

https://doi.org/10.33889/IJMEMS.2020.5.1.014

capture sound generated during a fall ( $\mathrm{Li}$ et al., 2011). By comparing the generated sound magnitude and direction (Popescu et al., 2008) with a specified threshold value, systems detect whether a fall has happened or not. One of the main advantages of these systems is that no physical contact is required with the system. Other advantages include high accuracy and easy and cheap installation. The main drawback of these systems is that once they are installed, person gets bound to the location where it is installed. Also, they are not applicable to a normal living environment because of the excessive noise generated by doing any normal work in the environment. These noises degrade the quality of the signal received by the sensor which can result in false fall detections.

\subsection{Vision based Fall Detection Systems}

Vision-based techniques monitors real-time movement of a person through a normal video camera (Cucchiara et al., 2007; Fu et al., 2008) or depth video camera (Shojaei-Hashemi et al., 2018) and in the background an algorithm is run in a dedicated PC to determine the posture of the person. As soon as a falling posture is detected by the algorithm, an alarm is generated to get help. Only a single setup of these systems can be used to supervise more than one person at a time. As compared to the previously mentioned systems, they are more convenient to use and are environment free. Also, a single video can gather more rich information than any other sensor installed in the environment. Another advantage of these systems is their versatility and robustness i.e. they can be employed in previously installed surveillance and security cameras (Liu et al., 2010). With the emergence of Deep Learning, computer vision has shown improvement in many operations such as object detection and recognition, image classification and segmentation. Researchers have now started using these deep learning concepts in many vision-based application areas, for example, to design a robust and intelligent vision-based fall detection system (Feng et al., 2014; Jokanovic et al., 2016c; Shojaei-Hashemi et al., 2018).

\section{Fall Detection Models using Machine Learning}

For classification between a fall and an ADL, Boyle and Karunanithi, (2008) and Chen et al. (2011) used threshold-based method (fall is detected when values of certain features cross their specified threshold), while others used predefined machine learning classifiers. Few surveys on thresholdbased fall detection techniques have already been done by researchers ( $\mathrm{Yu}, 2008$; Sposaro and Tyson, 2009; El-Bendary et al., 2013). In this paper, we have focused on a survey of fall detection models, developed using machine learning algorithms, as described below.

\subsection{Wearable Device based Fall Detection Systems}

Different researchers used a combination of sensors to design their model for human fall detection (Perry et al., 2009). Table 1 summarizes wearable systems which are using machine learning algorithms for classification purpose.

\subsubsection{Accelerometer}

Zhang et al. (2006) in their paper affixed tri-axial accelerometer to a belt and bound it around the waist of a human body. Thereafter, the collected acceleration data was sent to a one class Support Vector Machine (SVM) classifier to detect whether a fall has taken or not. Doukas et al. (2007) placed the accelerometer on the foot which will collect data generated by foot movement along the three axes (X, Y and Z). Support Vector Machine (SVM) classifier was then used to classify the foot movement in three classes i.e. walk, run and fall. Chen et al. (2010) used a tri-axial accelerometer to find acceleration along the three planes and plotted signal magnitude curve from them. Along with it, a fuzzy classifier was used at the end to detect and classify fall into different 
International Journal of Mathematical, Engineering and Management Sciences

Vol. 5, No. 1, 161-180, 2020

https://doi.org/10.33889/IJMEMS.2020.5.1.014

categories like falls following standing, falls following standing to sitting, etc. Chen et al. (2011) modified the work done in Chen et al. (2010), by including tilt angle along with acceleration. They used a tri-axial accelerometer and tiltmeter to find and analyze the human position. Tilt angle and acceleration values are then compared with their specific threshold values for final classification. Shan and Yuan (2010), also used the tri-axial accelerometer to generate feature vectors. These feature vectors are then passed to an SVM which classifies the activity in one of the two categories i.e. fall and an ADL. Verma et al. (2016) used two tri-axial accelerometers to collect acceleration data. One patch of the accelerometer was placed on the chest while another patch was placed on the thigh. Acceleration features extracted from each patch are passed to a multi-scale entropy (MSE) to obtain normalized MSE features which in the end provided as an input to the fuzzy logic model for prediction of posture condition. Lim et al. (2014) performed different experiments and concluded that a fall detection system works much better when the threshold-based method is combined with machine learning based method. They used a tri-axial accelerometer to read acceleration values along 3-axis. Extracted features are passed to three different models. Model 1 used a threshold-based method, model 2 used Hidden Markov model (HMM) while model 3 is a combination of model 1 and 2. In model 3, falls detected with the help of a simple threshold-based method are fed to the HMM.

Table 1. Wearable device-based Fall Detection Systems

\begin{tabular}{|c|c|c|c|}
\hline Author & Sensors & Features & Classifier \\
\hline Zhang et al. (2006) & Accelerometer & $\begin{array}{l}\text { Temporal and magnitude } \\
\text { of acceleration }\end{array}$ & SVM with One Class Classifier (accuracy 96.7\%) \\
\hline $\begin{array}{l}\text { Doukas et al. } \\
(2007)\end{array}$ & Accelerometer & $\begin{array}{l}\text { Acceleration along three } \\
\text { axis }\end{array}$ & SVM (accuracy - 98.2\%) \\
\hline Chen et al. (2010) & $\begin{array}{l}\text { Tri-Axial } \\
\text { Accelerometer }\end{array}$ & Acceleration vector & Fuzzy Rule Classifier \\
\hline \begin{tabular}{|l} 
Boyle and \\
Karunanithi (2008)
\end{tabular} & $\begin{array}{l}\text { Tri-Axial } \\
\text { Accelerometer }\end{array}$ & Angle, Acceleration vector & $\begin{array}{l}\text { Threshold based classifier (sensitivity - 95\%, specificity - } \\
100 \%)\end{array}$ \\
\hline $\begin{array}{l}\text { Shan and Yuan } \\
(2010)\end{array}$ & $\begin{array}{l}\text { Tri-Axial } \\
\text { Accelerometer }\end{array}$ & Acceleration vector & Support Vector Machine \\
\hline Lim et al. (2014) & $\begin{array}{l}\text { Tri-Axial } \\
\text { Accelerometer }\end{array}$ & Angle, Acceleration vector & $\begin{array}{l}\text { Threshold based classifier (sensitivity - 100\%, specificity } \\
91.56 \% \text {, accuracy - } 95.18 \% \text { )., HMM (sensitivity - } 99.17 \% \text {, } \\
\text { specificity } 99.69 \% \text {, accuracy - 95.89\%). Hybrid classifier } \\
\text { (sensitivity }-99.17 \% \text {, specificity } 99.69 \% \text {, accuracy }-99.5 \% \text { ) }\end{array}$ \\
\hline Verma et al. (2016) & $\begin{array}{l}\text { Tri-Axial } \\
\text { Accelerometer }\end{array}$ & $\begin{array}{l}\text { Acceleration vector, Multi } \\
\text { scale entropy features }\end{array}$ & Fuzzy Rule Classifier \\
\hline $\begin{array}{l}\text { Bourke and Lyons } \\
(2008)\end{array}$ & $\begin{array}{l}\text { Bi-Axial } \\
\text { Gyroscope }\end{array}$ & $\begin{array}{l}\text { Pitch role and Angular } \\
\text { velocity }\end{array}$ & Fuzzy Rule Classifier \\
\hline Li et al. (2009) & $\begin{array}{l}\text { Accelerometer, } \\
\text { Gyroscope }\end{array}$ & $\begin{array}{l}\text { Acceleration vector, Angular } \\
\text { velocity }\end{array}$ & Fuzzy Rule Classifier \\
\hline Zhao et al. (2018b) & $\begin{array}{l}\text { Tri-Axial } \\
\text { Gyroscope }\end{array}$ & Angular velocity along 3 -axis & $\begin{array}{l}\text { Decision tree (accuracy }-99.52 \% \text {, precision - 0.993, recall - } \\
0.995 \text { ) }\end{array}$ \\
\hline $\begin{array}{l}\text { Yuwono et al. } \\
(2012)\end{array}$ & Accelerometer & Acceleration & $\begin{array}{l}\text { Multi-layer perceptron, Augmented Radial Basis function, } \\
\text { Discrete Wavelet Transformation, Particle Swarm } \\
\text { Optimization (accuracy - 90.15\%) }\end{array}$ \\
\hline $\begin{array}{l}\text { Kerdegari et al. } \\
(2012)\end{array}$ & Accelerometer & $\begin{array}{l}\text { Acceleration, Position, } \\
\text { Angular velocity, Time }\end{array}$ & $\begin{array}{l}\text { Multi-layer perceptron (accuracy 90.15\%), SVM (accuracy } \\
\text { 86.68\%), Decision Tree (accuracy 84.28\%), Naive Bayes } \\
\text { (accuracy 88.57\%), ZeroR (accuracy 66.49\%) OneR } \\
\text { (accuracy } 73.19 \% \text { ) }\end{array}$ \\
\hline
\end{tabular}


International Journal of Mathematical, Engineering and Management Sciences

Vol. 5, No. 1, 161-180, 2020

https://doi.org/10.33889/IJMEMS.2020.5.1.014

\subsubsection{Gyroscope}

Bourke and Lyons (2008) used one bi-axial gyroscope to find pitch and roll angular velocity of a person. Thereafter these values are compared with a threshold value, if these values pass the thresholds then an alarm is triggered to warn of a fall. Li et al. (2009) in their work proposed an algorithm that used both accelerometers and gyroscopes. Along with the acceleration, the angular velocity was also used to detect the posture of a person. According to the transition between postures, the algorithm categorizes between a fall and a non-fall with the help of Fuzzy logic. Zhao et al. (2018b) in their paper proposed a method which uses a tri-axial gyroscope and placed it at the user's waist in order to get angular velocity along three axes. They divided the collected data into a set of consecutive and partially overlapping windows. Each of these windows is then fed to a decision tree for classification purpose. Experimental results have shown that our proposed method can effectively differentiate the fall events from other human daily activities despite of their high similarity in some cases, with the Accuracy of $99.52 \%$, Precision of 0.993 , Recall of 0.995 and Fmeasure of 0.994 .

Authors also provided comparative studies among different machine learning classifiers in order to find the best suitable classifier. Kerdegari et al. (2012) provided a comparative study of different machine learning classifiers used for classification of data generated by the accelerometer. They included algorithms like Naive Bayes, Multi-layer Perceptron (MLP), SVM, Decision tree, ZeroR, and OneR. In the end, they concluded that Multi-Layer Perceptron gives best results providing accuracy of $90.15 \%$. Yuwono et al. (2012) proposed a waist-band fall detection device. Their device used a tri-axial accelerometer for collecting movement data. If the magnitude of acceleration exceeds the specified threshold value, the acceleration signals were passed to a Gaussian distribution of Clustered Knowledge (GCK) signal generation module for feature extraction. This module first used a Discrete Wavelet Transform (DWT) for signal transformation and then used Regrouping Particle Swarm Optimization (RegPSO) for clustering. Thereafter, the neural network was used for classification. They also provided comparative analysis among classifiers like Augmented Radial Basis Function (ARBF) neural network and multi-layer perceptron (MLP). In their study, they found that their device performed more accurately when ARBF is combined with MLP.

\subsection{Context-Aware Based Fall Detection Systems}

Machine learning algorithms when applied to classify data generated by context-aware fall detection, provide a better result for scenarios where noise is less. A brief summary of the work done in this field is shown in Table 2 and 3.

\subsubsection{Ambient Based Fall Detection Systems}

A brief survey of ambient-based fall detection systems using machine learning algorithms is provided in Table 2.

\subsubsection{Floor Sensor}

Alwan et al. (2006) designed a fall detector which uses floor vibrations. The principle behind this model was based on the vibration pattern generated by a floor. It was seen that the floor vibration pattern during a fall differs with the vibration pattern generated during normal activities, like walking. Zigel et al. (2009) merged the concept of floor vibration pattern with audio sensed data to detect the actual location and frequency of fall. As output features like shock response spectrum and Mel Frequency Cepstral Coefficients (MFCC) were calculated and forwarded to Bayes

classifier to differentiate between a fall and an ADL. Rimminen et al. (2010) used a floor sensor 
International Journal of Mathematical, Engineering and Management Sciences

Vol. 5, No. 1, 161-180, 2020

https://doi.org/10.33889/IJMEMS.2020.5.1.014

with near-field imaging to find shape, size, and magnitude of vibration pattern and Bayesian filter for classification purpose.

Table 2. Ambient-based Fall Detection Systems

\begin{tabular}{|c|c|c|c|}
\hline Author & Sensors & Features & Classifier \\
\hline Alwan et al. (2006) & Piezoelectric Sensor & Floor vibration pattern & Threshold based \\
\hline Zigel et al. (2009) & Floor Sensor and Microphone & $\begin{array}{c}\text { Shock Response Spectrum, Mel Frequency } \\
\text { Cepstral Coefficient }\end{array}$ & Bayes Classification \\
\hline $\begin{array}{l}\text { Rimminen et al. } \\
\text { (2010) }\end{array}$ & $\begin{array}{l}\text { Floor Sensor and Near Field } \\
\text { Image Sensor }\end{array}$ & Position based on near field imaging floor & Bayesian Filter \\
\hline $\begin{array}{l}\text { Mastorakis and } \\
\text { Makris (2014) }\end{array}$ & KINECT Sensor & Position of a person (width, height, depth) & $\begin{array}{l}\text { Inactivity Detection in the } \\
\text { Postfall Phase }\end{array}$ \\
\hline Tzeng et al. (2010) & Pressure and Infrared Sensors & $\begin{array}{l}\text { Standard Deviation of Vertical Projection } \\
\text { Histogram, Standard Deviation of } \\
\text { Horizontal Projection Histogram, Aspect } \\
\text { Ratio }\end{array}$ & $\begin{array}{c}\text { Floor Pressure Signal } \\
\text { combined with infrared signal }\end{array}$ \\
\hline Toreyin et al. (2007) & Sound, Floor, Infrared Sensor & Wavelet based feature extraction & Hidden Markov Model \\
\hline Kim and Ling (2009) & Doppler Radar & Four-fold cross validation & SVM, Decision Tree \\
\hline $\begin{array}{l}\text { Jokanovic et al. } \\
\text { (2016a) }\end{array}$ & Doppler Radar & $\begin{array}{l}\text { Single Window Spectrogram to find time- } \\
\text { frequency motion data }\end{array}$ & $\begin{array}{c}\text { Softmax regression classifier } \\
\text { in DNN }\end{array}$ \\
\hline $\begin{array}{l}\text { Jokanović and Amin } \\
\text { (2017) }\end{array}$ & Range-Doppler Radars & $\begin{array}{l}\text { Spectrograms, Range maps to find time- } \\
\text { frequency motion data }\end{array}$ & $\begin{array}{c}\text { Logistic regression classifier } \\
\text { in DNN }\end{array}$ \\
\hline
\end{tabular}

\subsubsection{Infrared and Pressure Sensor}

Mastorakis and Makris (2014) used an infrared camera to observe human activity. First, the human is detected in a scene and a 3D bounding box is drawn around it. Then the velocity of human movement is calculated based on contraction or expansion of the 3D bounding box by measuring its width, height, and depth. Their detection system works on the idea that fall ends at an inactive state, i.e. after the fall, there is no movement. Hence, calculated velocity value is compared to a specific threshold value in order to detect a fall. Tzeng et al. (2010) used an infrared camera in order to observe the area whenever the installed floor pressure sensor detects any pressure. The pressure sensor used a linear variable differential transformer to detect human activity which then signals the camera to take photographic records. Then, smoothing and filtering were applied on captured images for clear feature extraction. These extracted features combined with pressure threshold value judge whether a fall has taken or not. In the method proposed by Toreyin et al. (2007), different types of context-aware sensors are used together (floor, infrared, sound) to collect data. This data is then passed to a Wavelet based feature extraction system to extract features. In the end, these extracted features are passed through HMM for classification.

\subsubsection{Doppler Radar}

Kim and Ling (2009) used Doppler Radar and Doppler Spectrogram for extracting features from seven different activities, i.e. walking, running, crawling, sitting still, boxing, walking with a stick, boxing while standing in one place and boxing while moving forward. Extracted features are then examined with the help of short-time Fourier transform (STFT) to find Doppler signatures. Then, a four fold-class validation is applied for optimization of features. In the end, optimized features 
International Journal of Mathematical, Engineering and Management Sciences

Vol. 5, No. 1, 161-180, 2020

https://doi.org/10.33889/IJMEMS.2020.5.1.014

are passed to SVM and Decision-Tree for classification. Jokanovic et al. (2016a) used SingleWindow Spectrogram (SWS) to collect data. Afterwards they applied Time-Frequency analysis (TF) on collected data to learn about the velocity components of human body parts. Extracted features are then passed to DNN formed from Stack Auto-Encoder and Softmax Regression Classifier. In their experiment, they have shown that their model outperforms other PCA (Principal Component Analysis) based model provided by them in Jokanovic et al. (2016b). Jokanovic and Amin (2017), used Range-Doppler Radars to collect time-frequency data and range data. DNN is designed consisting of two stack sparse auto-encoders and linear regression classifier for training and classification.

Researchers not only deployed sensors on the floor to detect human activity but also developed a special type of sensor floor (Klack et al., 2010). This sensor floor monitors human position within that room where it is deployed. It can detect abnormal patterns in human activity and activate rescue procedure in case of emergency events like fall. This sensor floor is quite useful for scenarios where elderly people live independently. But this installation will become quite expensive for shelter homes for the elderly care and hospitals.

\subsubsection{Acoustic Based Fall Detection Systems}

Table 3 provides a brief survey of acoustic-based fall detection systems which use machine learning algorithms for the design of their classifier.

Popescu et al. (2008) designed an acoustic fall detection system (acoustic-FADE). This system uses a linear array of 3 microphones to collect sound data generated when a person falls. This system detects a fall by comparing and analysing the height of the sound sensor from the ground with the size of the generated sound. If the sound is located at a height of more than 2 feet then it is considered as a false alarm. In their experiment, they had shown that their system generates 0 error rate when the fall detection rate is $70 \%$ and generates 5 false fall detections per hour when fall detection rate is $100 \%$. Li et al. (2012) used circular microphone array. With the help of phase transform technique, a 3D estimation of sound source location is generated. Thereafter, the signal is enhanced and the Mel Frequency Cepstral Coefficient (MFCC) features are extracted from them. In the end, Nearest-Neighbour classifier is used to classify the sound in two classes i.e. fall and non-fall. Zhuang et al. (2009) developed a model which uses GMM super vectors for modeling sound segments. After that, an SVM is built on GMM super vectors to classify sound segments into fall and various other types of noise. As compared to standard GMM classifier, their model improves the accuracy of the fall detection system from $37 \%$ to $41 \%$ by re-classifying confused labels generated by GMM. Work done by Popescu et al. (2008) is again modified by Li et al. (2010), where they used a circular array of 8 microphones to provide 3D estimation of sound source location. Thereafter, KNN classifier is used with a value of $\mathrm{K}=1$. When height is not included, their system detects $100 \%$ of the falls with 30 false alarms. And when the height feature is used, their system generates 5 false alarms per hour. Li et al. (2011) designed a system consisting of a circular array of 8 microphones in order to find the sound source location. A Hamming window function is computed to find the time window during which sound was generated and then a time reference is added to that window. Then a time reference is selected where the amplitude is strongest. For classification, the Mel Frequency Cepstral Coefficient (MFCC) is calculated and K-Nearest Neighbor (KNN) is applied to it. 
International Journal of Mathematical, Engineering and Management Sciences

Vol. 5, No. 1, 161-180, 2020

https://doi.org/10.33889/IJMEMS.2020.5.1.014

Table 3. Acoustic-based fall detection systems

\begin{tabular}{|l|l|l|l|}
\hline \multicolumn{1}{|c|}{ Author } & \multicolumn{1}{|c|}{ Sensors } & \multicolumn{1}{|c|}{ Features } & \multicolumn{1}{|c|}{ Classifier } \\
\hline Popescu et al. (2008) & $\begin{array}{l}\text { Linear Array of } 3 \\
\text { Microphones }\end{array}$ & Loudness and height of the sound & Defined their own classifier \\
\hline Li et al. (2012) & Microphone Array & $\begin{array}{l}\text { Mel Frequency Cepstral Coefficient, source } \\
\text { location }\end{array}$ & K-Nearest Neighbor classifier \\
\hline Zhuang et al. (2009) & $\begin{array}{l}\text { Single far-field } \\
\text { microphone }\end{array}$ & $\begin{array}{l}\text { Pairwise difference between sound segments using } \\
\text { Euclidean Distance }\end{array}$ & $\begin{array}{l}\text { Gaussian Mixture Model with } \\
\text { Support Vector Machine }\end{array}$ \\
\hline Li et al. (2010) & $\begin{array}{l}\text { Circular Microphone } \\
\text { Array }\end{array}$ & $\begin{array}{l}\text { Mel Frequency Cepstral Coefficient, sound } \\
\text { location }\end{array}$ & K-Nearest Neighbor classifier \\
\hline Li et al. (2011) & $\begin{array}{l}\text { Circular Array of 8 8 } \\
\text { Microphone }\end{array}$ & $\begin{array}{l}\text { 3-D estimation of the sound, the height of the } \\
\text { sound, Adaptive Signal Windowing }\end{array}$ & K-Nearest Neighbor classifier \\
\hline
\end{tabular}

\subsection{Vision-Based Fall Detection Systems}

In the present scenario, we can see that the surveillance cameras are present everywhere. Besides the security purpose, these surveillance cameras can also be used for other different purposes including human fall detection. With the advancement in Machine Learning technology, intelligent vision-based fall detection systems are being developed by using different cameras in our daily life. Table 4 to 6 provides a summary of vision-based fall detection systems which use machine learning algorithms.

Fu et al. (2008) developed a human fall detection system by using an asynchronous temporal contrast vision sensor. The method reports change in any temporal contrast by extracting the change in pixel values. These pixel values are then processed in a PC to construct human silhouette. The motion of the centre of a silhouette is computed at the end which is used for classification. Cucchiara et al. (2007) designed a multi-camera system which extracts the human body from a scene by applying background suppression on scene images with selective and adaptive updates. After human extraction, they applied their proposed probabilistic tracking algorithm to track human movement. For detection of a human fall movement, they proposed a method based on hidden Markov model (HMM). In Vishwakarma et al. (2007) proposed a model which consist of three phases. The work of phase one is to detect and track human present in a scene with the help of the background subtraction method. A bounding box is drawn around the human. Different features like horizontal and vertical gradient values, aspect ratio and fall angle are returned as an output of phase 1 and forwarded to phase 2 . The second phase is a fall detection phase, which uses the help of horizontal and vertical gradient values and aspect ratio to detect a fall. Once a fall is detected, fall confirmation is done in phase 3 with the help of fall angle with respect to a horizontal axis of the drawn bounding box. 
International Journal of Mathematical, Engineering and Management Sciences

Vol. 5, No. 1, 161-180, 2020

https://doi.org/10.33889/IJMEMS.2020.5.1.014

Table 4. Vision-based fall detection systems Part-1

\begin{tabular}{|c|c|c|c|c|}
\hline Author & Sensors/Technique & Features & Classifier & Accuracy \\
\hline Fu et al. (2008) & Contrast Vision Sensor & Change in illumination, motion vector & $\begin{array}{l}\text { Threshold based } \\
\text { classifier }\end{array}$ & AC $-84 \%$ \\
\hline \begin{tabular}{|l} 
Cucchiara et al. \\
$(2007)$
\end{tabular} & $\begin{array}{l}\text { Multi-camera vision system with } \\
\text { background suppression }\end{array}$ & $\begin{array}{l}\text { Geometrical and color features together } \\
\text { with the projection of the silhouettes shape } \\
\text { on X and Y axis }\end{array}$ & $\begin{array}{l}\text { Hidden Markov } \\
\text { Model (HMM) }\end{array}$ & - \\
\hline $\begin{array}{l}\text { Vishwakarma et } \\
\text { al. (2007) }\end{array}$ & $\begin{array}{l}\text { Background subtraction with } \\
\text { Gaussian mixture model }\end{array}$ & $\begin{array}{l}\text { Aspect ratio, horizontal and vertical } \\
\text { gradient distribution in X-Y plane and fall } \\
\text { angle }\end{array}$ & Finite state machine & - \\
\hline $\begin{array}{l}\text { Hazelhoff } \\
\text { Han (2008) }\end{array}$ & $\begin{array}{l}\text { Background subtraction, tracking } \\
\text { algorithm to filter person position }\end{array}$ & $\begin{array}{l}\text { Orientation of person from main axis, } \\
\text { Ratio of variance in horizontal and vertical } \\
\text { direction, Skin color }\end{array}$ & $\begin{array}{ll}\text { Multi } & \text { Frame } \\
\text { Gaussian } & \end{array}$ & SE-100\% \\
\hline $\begin{array}{l}\text { Foroughi } \\
(2008 \mathrm{a})\end{array}$ & Silhouette and head movement & $\begin{array}{l}\text { Projection histograms of the segmented } \\
\text { silhouette }\end{array}$ & $\begin{array}{l}\text { Multi layer } \\
\text { perceptron NN }\end{array}$ & $\begin{array}{l}\text { SE } 92.8 \% \\
\text { SP } 97.6 \%\end{array}$ \\
\hline $\begin{array}{l}\text { Foroughi } \\
(2008 \mathrm{~b})\end{array}$ et al. & $\begin{array}{l}\text { Eigen-space approach for motion } \\
\text { recognition }\end{array}$ & Integrated time motion images & Neural Network & AC $-91 \%$ \\
\hline \begin{tabular}{|l} 
Anderson et al. \\
$(2009)$
\end{tabular} & Multi-camera vision system & $\begin{array}{l}\text { Finding silhouette centroid, height and } \\
\text { body orientation of person }\end{array}$ & $\begin{array}{l}\text { Two Level Fuzzy } \\
\text { Logic }\end{array}$ & $\begin{array}{l}\text { SE - } 100 \% \\
\text { SP - } 93 \%\end{array}$ \\
\hline $\begin{array}{l}\text { Willems } \\
(2009)\end{array} \quad$ et $\quad$ al. & $\begin{array}{l}\text { Gray scale processing algorithm, } \\
\text { background subtraction }\end{array}$ & $\begin{array}{l}\text { Ellipse is drawn around the object aspect } \\
\text { ratio and fall angle }\end{array}$ & $\begin{array}{l}\text { Vertical Projection } \\
\text { Histogram }\end{array}$ & AC $-80 \%$ \\
\hline Liu et al. (2010) & Frame Difference & $\begin{array}{l}\text { Ratio and difference of human silhouette } \\
\text { bounding box height and width }\end{array}$ & K-NN & AC $-84.4 \%$ \\
\hline $\begin{array}{l}\text { Rougier } \\
(2011)\end{array}$ & \begin{tabular}{|l} 
Background subtraction and \\
analysis of human shape \\
deformation
\end{tabular} & End points from the silhouette & $\begin{array}{l}\text { Gaussian } \text { Mixture } \\
\text { Model }\end{array}$ & AC $-98 \%$ \\
\hline $\begin{array}{l}\text { Krekovic et al. } \\
(2012)\end{array}$ & Background Subtractor MOG2 & MHI and shape (ellipse) deformation & Fuzzy rule & AC $-90 \%$ \\
\hline
\end{tabular}

Hazelhoff and Han (2008) in their model deployed two perpendicular cameras for extraction of the foreground region. For each extracted foreground, they applied principal component analysis in order to find the direction of the main axis of human body and its variance in $\mathrm{x}$ and $\mathrm{y}$ plane. These extracted features are then fed into a multi-frame Gaussian classifier for recognition of fall event. Foroughi et al. (2008a) detected fall from a video sequence by applying shape change analysis on it with the help of approximated ellipse drawn around the person's body present in the video frame. Projection histograms are then evaluated to find the change in the head position. The resultant extracted features are then passed to a Multi-layer Perceptron Neural Network for classification. In Foroughi et al. (2008b) proposed another work to detect movement patterns from a video sequence in the form of motion images. In these images, along with the occurrence of motion, motion information and timestamp are also stored, hence, called integrated time motion images (ITMI). Eigen-space approach is then applied to these images for feature reduction. The result of this phase is then passed to a multi-layer perceptron neural network designed for Motion Recognition and classification.

Anderson et al. (2009) defined the activity of fall detection method in two levels. The job of the first level is to extract 3D human silhouette present in a scene with the help of multiple cameras. This 3D human silhouette is called voxel person. Fuzzy logic is used to perform linguistic summarization of state of voxel person in the second level. With the help of summarization and 
International Journal of Mathematical, Engineering and Management Sciences

Vol. 5, No. 1, 161-180, 2020

https://doi.org/10.33889/IJMEMS.2020.5.1.014

activity interference, a fall is detected. Willems et al. (2009) used a background subtraction method to differentiate a person from their background in a video frame. Once detected, an ellipse is drawn around it to calculate aspect ratio and fall angle. Calculated features are then used to design vertical projection histogram which in the end confirms the fall.

Liu et al. (2010) also generated human silhouette from a scene and drawn a bounding box around it. After then vertical projection histogram for the silhouette is generated. Generated histogram and ratio of the difference of width and height of the bounding box are then fed into a KNN classifier for fall detection. Rougier et al. (2011) developed a system known as the Human Shape Deformation Analysis. In their system, they used a shape matching technique to keep track of a person's silhouette. According to them, an increase in the deformation of silhouette represents a fall. Four cameras are used to analyze the body shape of the person. In the end, three component GMM classifier is proposed which uses two different features, i.e. full Procrustes distance features and mean matching cost features to classify recorded activity. Krekovic et al. (2012) used background subtractor MOG2 algorithm present in the OpenCV library for motion analysis and object tracking. Once a person's body is tracked, an ellipse is drawn around it. Then Motion History Image Coefficient (MHIC) is calculated to find the amount of movement done in the two consecutive frames. In the final step, body shape analysis and classification is done by checking the changes in the horizontal and vertical axis of that ellipse.

Table 5. Vision-based fall detection systems Part-2

\begin{tabular}{|c|c|c|c|c|}
\hline Authors & Sensors & Features & Classifier & Accuracy \\
\hline $\begin{array}{l}\text { Feng } \\
(2014)\end{array}$ et al. & Background subtraction & $\begin{array}{l}\text { Binary image with human as } \\
\text { foreground }\end{array}$ & $\begin{array}{l}\text { Restricted Boltzman } \\
\text { machines (RBM), SVM, } \\
\text { Deep belief network } \\
(\mathrm{DBN})\end{array}$ & $\begin{array}{l}\text { RBM } 86 \% \\
\text { DBN-84.3\% } \\
\text { SVM-81.7\% }\end{array}$ \\
\hline $\begin{array}{l}\text { Charfi et al. } \\
(2012)\end{array}$ & $\begin{array}{l}\text { Human silhouette movement } \\
\text { tracking }\end{array}$ & $\begin{array}{l}\text { Width and height of bounding } \\
\text { box, human trajectory and } \\
\text { projection histogram }\end{array}$ & SVM & $\begin{array}{l}\text { RE }-98 \% \\
\text { SP }-99.6 \% \\
P R-94.2 \%\end{array}$ \\
\hline $\begin{array}{l}\text { Charfi et al. } \\
(2013)\end{array}$ & 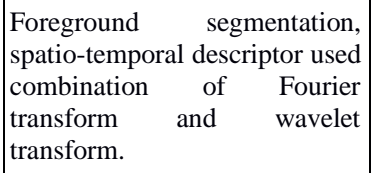 & $\begin{array}{l}\text { Aspect ratio, ellipse } \\
\text { orientation and movement }\end{array}$ & SVM, Adaboos & $\begin{array}{l}\text { SVM (SP }-99.69 \%, \mathrm{AC}- \\
99.61 \%, \mathrm{PR}-94.23 \%) \\
\text { Adaboost }(\mathrm{SP}-99.97 \% \text {, } \\
\text { AC - 99.42\%, PR - } \\
95.91 \%)\end{array}$ \\
\hline $\begin{array}{l}\text { Jokanovic et al. } \\
(2016 \mathrm{c})\end{array}$ & Multi Window Spectrogram & Time-frequency motion data & $\begin{array}{l}\text { Softmax Regression } \\
\text { Classifier in DNN }\end{array}$ & AC $-90 \%$ \\
\hline $\begin{array}{l}\text { Jokanovic et al. } \\
(2016 b)\end{array}$ & Principle Component Analysis & $\begin{array}{l}\text { Vectorized version of gray } \\
\text { scale spectrogram }\end{array}$ & $\begin{array}{l}\text { Minimum Euclidean } \\
\text { Distance Classifier }\end{array}$ & AC $-83 \%$ \\
\hline $\begin{array}{l}\text { Zhi and Hui } \\
(2017)\end{array}$ & $\begin{array}{l}\text { Background } \quad \text { interference } \\
\text { elimination }\end{array}$ & $\begin{array}{l}\text { Ellipse is drawn around the } \\
\text { object }\end{array}$ & 3D-CNN & AC $-96 \%$ \\
\hline $\begin{array}{l}\text { de Miguel et al. } \\
(2017)\end{array}$ & $\begin{array}{l}\text { Background } \quad \text { subtraction, } \\
\text { Kalman filter }\end{array}$ & $\begin{array}{l}\text { Aspect ratio, speed change, } \\
\text { angle }\end{array}$ & $\mathrm{K}-\mathrm{NN}$ & $\begin{array}{l}\text { SE }-96 \% \\
\text { SP }-97 \%\end{array}$ \\
\hline Lu et al. (2017) & 3-D CNN with LSTM & Automatic feature extraction & SVM & AC $-99.73 \%$ \\
\hline
\end{tabular}

Feng et al. (2014) also used the background subtraction method to track a human body. After then they used two deep neural networks i.e. deep belief networks (DBN) and restricted Boltzmann machines (RBM) for classification. They also compared the output of their deep network models 
International Journal of Mathematical, Engineering and Management Sciences

Vol. 5, No. 1, 161-180, 2020

https://doi.org/10.33889/IJMEMS.2020.5.1.014

with SVM and concluded that deep networks perform better than SVM. In Charfi et al. (2012), they worked on the human silhouette. Features like width and height of bounding box, human trajectory, and projection histogram are extracted by tracking the movement of bounding box drawn around the human silhouette. These extracted features are then fed to an SVM classifier. Charfi et al. (2013) proposed another work on human fall detection. This work was based on foreground segmentation method to extract features like coordinates of bounding box drawn around the human, its aspect ratio, ellipse orientation, and movement. They proposed their own spatiotemporal descriptor named STHF which used a combination of Fourier transform and wavelet transform. They used this descriptor for feature transformation of extracted geometrical features. For classification purposes, they provided a comparative analysis between SVM and Adaboost.

Jokanovic et al. (2016c) modified their previous work done in Jokanovic et al. (2016a) by using Multi-Window Spectrogram (MWS) for data collection. They provided a comparative study of different TF signal representations, i.e. SWS, MWS, Wavelet Transform, and Wigner-Ville Distribution. In result, they have shown that MWS outperforms all the three mentioned TF analyzers. Jokanovic et al. (2016c) developed two models based on Principal Component Analysis (PCA). Step one is the same for both the models. In step one, SWS is used to read human motions and generates output in the form of MicroDoppler Signatures. Step two for model one uses three eigen images. While step two for the second model uses eigen space generated for each class. In the final step, Minimum Euclidean Distance Classifier is used to find the class with the closest match. Zhi and Hui (2017) designed a method known as Two-Stream CNN. One stream is made of 3-Dimensional $\mathrm{CNN}$ to eliminate the interference produced by video background. The second stream is made of VGGNet-16 CNN to find optical flow in video frames. Finally, the softmax function is applied to the output of both the streams for finding the final result.

In de Miguel et al. (2017), they divided the work into a few steps. In the first step, background subtraction is used to remove uninteresting objects. The output is then passed to Kalman filter for the next step, i.e. noise removal and to keep track of objects present in the scene. After then output images are passed through the optical flow to eliminate static objects present in the scene. Extracted features are then passed through a K-Nearest Neighbour classifier for classification. Lu et al. (2017) designed a visual attention based 3-Dimensional CNN fall detection system. The system uses kinect data and passes it to Automatic Feature Extractor module for its training. Once trained, features are extracted from testing data, an LSTM based visual attention scheme is used to focus on specific regions. They trained their model on Multiple Cameras Fall Dataset.

Nunez-Marcos et al. (2017) used optical flow images which represent the motion of two consecutive frames. A set of optical flow images is stacked and passed to a CNN for feature extraction. In the end, a fully-connected neural network is used for classification purpose. Harrou et al. (2017) proposed a variation of multivariate exponentially weighted moving average monitoring scheme (MEWMA). MEWMA charts generated by this scheme are then used to detect an anomaly in a scene. In the end, they used SVM classifier to differentiate between true fall event and other fall-like events. They also provided a comparative study of their work with other machine learning algorithms like naive Bayes, neural network and KNN.

Ge et al. (2017) proposed a method which uses two steam CNN input, one for spatial and one for temporal features. Spatial features are extracted by computing RGB difference between two consecutive frames while temporal features are extracted by using a temporal optical flow of video frames. Sparse representation framework with residual-based pooling is then applied on CNN 
International Journal of Mathematical, Engineering and Management Sciences

Vol. 5, No. 1, 161-180, 2020

https://doi.org/10.33889/IJMEMS.2020.5.1.014

extracted features to obtain sparse feature codes. In order to capture the sequential information in a video, long-range temporal feature representation is considered by concatenating it with sparse feature codes which are then provided as an input to SVM classifier for classification purpose. Fan et al. (2017) used background subtraction technique to extract the human body from a scene and enclosed it in an ellipse. Then, multi-directional statistical analysis is done by developing a normalized directional histogram around the center of a drawn ellipse. This normalized histogram in result generates 12 static and 8 dynamic features. In the end, resultant features are provided as an input to a directed acyclic graph SVM to classify human postures like sitting, lying, crouching and standing. By counting the number of occurrences of lying postures in a short temporal window, a fall can be detected.

Table 6. Vision-based fall detection systems Part-3

\begin{tabular}{|c|c|c|c|c|}
\hline Authors & Sensors & Features & Classifier & Accuracy \\
\hline $\begin{array}{l}\text { Nunez-Marcos } \\
\text { et al. (2017) }\end{array}$ & Optical flow of images & $\begin{array}{l}\text { Displacement vector field in } \\
\text { two consecutive frames }\end{array}$ & $\mathrm{CNN}$ & $\begin{array}{l}\text { SP }-97.23 \% \\
\text { SE }-93.47 \%\end{array}$ \\
\hline $\begin{array}{l}\text { Harrou et al. } \\
(2017)\end{array}$ & $\begin{array}{lr}\text { Multivariate exponentially } \\
\text { weighted moving } \\
\text { monitoring } & \text { scheme } \\
\text { (MEWMA) } & \end{array}$ & MEWMA charts & $\begin{array}{l}\text { SVM, NN, nave Bayes, K- } \\
\text { NN }\end{array}$ & $\begin{array}{l}\text { KNN ( AC 91.94\%, } \\
\text { SP 86\%, PR 83\%) } \\
\text { NN (AC 95.15\%, } \\
\text { SP 91\%, PR 90.3\%) } \\
\text { Naive Bayes (AC 93.55\%, } \\
\text { SP - 88.6\%, } \\
\text { PR 87\%) } \\
\text { MEWMA-SVM } \\
\text { (AC 96.66\%, SP 94.93\%, } \\
\text { PR 93.55\%) }\end{array}$ \\
\hline Ge et al. (2017) & $\begin{array}{l}\text { Residual based pooling on } \\
\text { two stream CNN }\end{array}$ & $\begin{array}{l}\text { RGB difference between two } \\
\text { consecutive frames }\end{array}$ & SVM & AC $-90.50 \%$ \\
\hline $\begin{array}{l}\text { Fan et al. } \\
(2017)\end{array}$ & $\begin{array}{l}\text { Background subtraction, } \\
\text { multi-directional statistical } \\
\text { analysis }\end{array}$ & $\begin{array}{l}\text { Normalized } \\
\text { histogram }\end{array}$ & $\begin{array}{lr}\text { Directed Acyclic } & \text { Graph } \\
\text { SVM (DAGSVM), } & \text { Multi- } \\
\text { layer perceptron } & \text { NN } \\
(\text { MLPNN) } & \\
\end{array}$ & $\begin{array}{l}\text { MLPNN } \\
(\text { AC }-92.9 \%) \\
\text { DAGSVM } \\
(\text { AC }-97.1 \%)\end{array}$ \\
\hline $\begin{array}{l}\text { Hsieh and Jeng } \\
(2017)\end{array}$ & $\begin{array}{l}\text { Feature feedbackm } \\
\text { mechanism scheme }\end{array}$ & $\begin{array}{l}\text { Statistic Euclidean distance of } \\
\text { histogram }\end{array}$ & Optical flow feedback CNN & $\begin{array}{l}\text { Not specific } \\
\text { to fall }\end{array}$ \\
\hline $\begin{array}{l}\operatorname{Min} \text { et al. } \\
(2018)\end{array}$ & $\begin{array}{l}\text { Faster R-CNN, Activity } \\
\text { characteristic extraction }\end{array}$ & $\begin{array}{l}\text { Space relation, aspect ratio, } \\
\text { aspect ratio velocity, centroid, } \\
\text { motion velocity }\end{array}$ & $\begin{array}{l}\text { Threshold } \\
\text { classification }\end{array}$ & $\begin{array}{l}\text { AC }-94.5 \% \\
\text { PR }-94.4 \% \\
\text { RE }-94.9 \%\end{array}$ \\
\hline $\begin{array}{l}\text { Lie et al. } \\
(2018)\end{array}$ & CNN, LSTM & 2D skeletons & RNN & AC $-88.9 \%$ \\
\hline $\begin{array}{l}\text { Anishchenko } \\
\text { et al. (2018) }\end{array}$ & Modified AlexNet CNN & Cohens kappa & $\mathrm{CNN}$ & AC $-99 \%$ \\
\hline $\begin{array}{l}\text { Zhao et al. } \\
\text { (2018a) }\end{array}$ & $\begin{array}{l}\text { Depth camera for visual, } \\
\text { Random Forest for human } \\
\text { body detection }\end{array}$ & Motion feature Space & Large Margin based KNN & $\begin{array}{l}\text { SE }-86 \% \\
\text { SP }-98 \%\end{array}$ \\
\hline
\end{tabular}

Min et al. (2018) designed a method for detecting falls on furniture. In the first step, human and furniture are detected in the scene (video frame). For this purpose, they have used Faster R-CNN. With the help of the position of human and furniture, space relation is found between them. By measuring changes in other features like human aspect ratio, velocity, centroid and speed of motion along with space relation, a fall can be detected. Lie et al. (2018) used CNN to find 2D skeletons 
International Journal of Mathematical, Engineering and Management Sciences

Vol. 5, No. 1, 161-180, 2020

https://doi.org/10.33889/IJMEMS.2020.5.1.014

of human from each video frame. The output is then passed through a Recurrent Neural Network (RNN) with LSTM to process this series of skeletons by finding spatial as well as temporal information from them and for classification. Anishchenko (2018) modified the pre-trained AlexNet CNN architecture for two-class classification (fall and non-fall). Transfer learning technique is used to train their modified model.

Yang et al. (2016) used Kinect sensor to capture 3D depth images of the scene. Pre-processing of these images is done by applying a median filter and background subtraction which in result generates moving human silhouette in consecutive depth images. An ellipse is drawn around the human silhouette. Horizontal and vertical projection histograms are then obtained from a disparity map of depth images. These disparity maps are then used to obtain a floor plane equation. For classification purposes, they used a threshold-based method where the angle between centroid of human silhouette and floor plane is compared to a threshold value for fall detection and classification. Zerrouki and Houacine (2018) proposed a method which applied curvelet transform on background subtracted video frames. The resultant curvelet coefficients are then used as attributes of a human silhouette. A feature selection technique is then applied to these features. This technique was based on differential evolution (DE). The reduced feature vector was then fed to an SVM classifier for posture classification. In the end, HMM is used to recognize human activity i.e. fall and non-fall. They used a depth camera to detect fall from the bed. Depth camera output is passed to a Random Forest algorithm for detection of the human body by extracting human head and upper body center. Then after detection of upper-body motion, Large Margin Nearest Neighbour classifier is used for two-class classification (fall and non-fall).

\section{Conclusion}

This paper provides a literature review of different approaches used for the development of an automatic human fall detection system. Three different technologies are used: (i) wearable based device, (ii) context-aware fall detection systems and (iii) vision based systems. The underlying methods for each previously mentioned category along with a description of their algorithm and performance statistics are also described in detail.

Due to the advancement in technologies, intelligent systems have been developed with the help of Machine Learning. Whether it is a wearable device, context-aware device or any vision-based device, machine learning algorithms have been merged with them to make them more intelligent and independent. We have focused our direction towards the use of different machine learning algorithms for the development of intelligent automatic human fall detection system. We have provided a brief review of different machine learning algorithms used with the existing technologies.

From the above survey, we can see that most of the recent research is focused on the use of vision based technology for the development of a robust fall detection system. There are some limitations to using wearable based technology and context-aware based technology. In case of wearable device-based method, people often forget to carry such devices with them and if the system is button operated, they forget to press the button after fall to generate an alarm. While children often use this button just to create inconvenience for their parents. While context aware based methods use audio, pressure and vibration data generated during a fall which is prone to environmental noise.

In comparison to vision based approaches, vision based systems can overcome the above mentioned drawbacks. Also, surveillance cameras have been installed everywhere in the environment such as 
International Journal of Mathematical, Engineering and Management Sciences

Vol. 5, No. 1, 161-180, 2020

https://doi.org/10.33889/IJMEMS.2020.5.1.014

hospitals, residence, shelter homes for children and elder, etc. They gather data which has rich information which can be utilized for fulfilling several functions. Also, with the introduction of Deep Learning, working on images and videos have become easy and smart. Hence, we can say that for the development of future systems, vision-based technology should be mainly used.

\section{Conflict of Interest}

The authors declare that there is no conflict of interest for this publication.

\section{Acknowledgement}

The authors express their gratitude to the reviewers for their valuable suggestions towards the improvement in the quality of the paper.

\section{References}

Abbate, S., Avvenuti, M., Corsini, P., Vecchio, A., \& Light, J. (2010). Monitoring of human movements for fall detection and activities recognition in elderly care using wireless sensor network: a survey. Wireless Sensor Networks: Application-Centric Design. IntechOpen, pp. 147-166.

Alwan, M., Rajendran, P.J., Kell, S., Mack, D., Dalal, S., Wolfe, M., \& Felder, R. (2006, April). A smart and passive floor-vibration based fall detector for elderly. In 2006 2nd International Conference on Information \& Communication Technologies (Vol. 1, No. 2, pp. 1003-1007). IEEE. Damascus, Syria.

Anderson, D., Luke, R.H., Keller, J. M., Skubic, M., Rantz, M., \& Aud, M. (2009). Linguistic summarization of video for fall detection using voxel person and fuzzy logic. Computer Vision and Image Understanding, 113(1), 80-89.

Anishchenko, L. (2018, May). Machine learning in video surveillance for fall detection. In 2018 Ural Symposium on Biomedical Engineering, Radioelectronics and Information Technology (USBEREIT) (pp. 99-102). IEEE. Yekaterinburg.

Bourke, A.K., \& Lyons, G.M. (2008). A threshold-based fall-detection algorithm using a bi-axial gyroscope sensor. Medical Engineering \& Physics, 30(1), 84-90.

Boyle, J., \& Karunanithi, M. (2008, August). Simulated fall detection via accelerometers. In 2008 30th Annual International Conference of the IEEE Engineering in Medicine and Biology Society (pp. 12741277). IEEE. Vancouver, BC, Canada.

Charfi, I., Miteran, J., Dubois, J., Atri, M., \& Tourki, R. (2012, November). Definition and performance evaluation of a robust SVM based fall detection solution. In 2012 Eighth International Conference on Signal Image Technology and Internet Based Systems (pp. 218-224). IEEE. Sorrento-Naples, Italy.

Charfi, I., Miteran, J., Dubois, J., Atri, M., \& Tourki, R. (2013). Optimized spatio-temporal descriptors for real-time fall detection: comparison of support vector machine and Adaboost-based classification. Journal of Electronic Imaging, 22(4), 041106.

Chen, D., Feng, W., Zhang, Y., Li, X., \& Wang, T. (2011, December). A wearable wireless fall detection system with accelerators. In 2011 IEEE International Conference on Robotics and Biomimetics (pp. 2259-2263). IEEE. Phuket, Thailand.

Chen, G.C., Huang, C.N., Chiang, C.Y., Hsieh, C.J., \& Chan, C.T. (2010, June). A reliable fall detection system based on wearable sensor and signal magnitude area for elderly residents. In International Conference on Smart Homes and Health Telematics (pp. 267-270). Springer. Berlin, Heidelberg. 
International Journal of Mathematical, Engineering and Management Sciences

Vol. 5, No. 1, 161-180, 2020

https://doi.org/10.33889/IJMEMS.2020.5.1.014

Cucchiara, R., Prati, A., \& Vezzani, R. (2007). A multi-camera vision system for fall detection and alarm generation. Expert Systems, 24(5), 334-345.

de Miguel, K., Brunete, A., Hernando, M., \& Gambao, E. (2017). Home camera-based fall detection system for the elderly. Sensors, 17(12), 2864.

Doukas, C., Maglogiannis, I., Tragas, P., Liapis, D., \& Yovanof, G. (2007, September). Patient fall detection using support vector machines. In IFIP International Conference on Artificial Intelligence Applications and Innovations: Artificial Intelligence and Innovations 2007: from Theory to Applications. AIAI 2007. IFIP the International Federation for Information Processing (Vol. 247 pp. 147-156). Springer. Boston, MA.

Duque, G. (2016) Age-related physical and physiologic changes and comorbidities in older people: association with falls. In: Huang A., Mallet L. (eds) Medication-Related Falls in Older People (pp. 6773). Adis, Cham.

El-Bendary, N., Tan, Q., Pivot, F.C., \& Lam, A. (2013). Fall detection and prevention for the elderly: a review of trends and challenges. International Journal on Smart Sensing \& Intelligent Systems, 6(3), 1230-1266.

Fan, K., Wang, P., Hu, Y., \& Dou, B. (2017). Fall detection via human posture representation and support vector machine. International Journal of Distributed Sensor Networks, 13(5), 1-21. https://doi.org/10.1177/1550147717707418.

Feng, P., Yu, M., Naqvi, S.M., \& Chambers, J.A. (2014, August). Deep learning for posture analysis in fall detection. In 2014 19th International Conference on Digital Signal Processing (pp. 12-17). IEEE. Vancouver, Canada.

Foroughi, H., Aski, B.S., \& Pourreza, H. (2008a, December). Intelligent video surveillance for monitoring fall detection of elderly in home environments. In 2008 11th international conference on computer and information technology (pp. 219-224). IEEE. Piscataway, New Jersey.

Foroughi, H., Naseri, A., Saberi, A., \& Yazdi, H.S. (2008b, October). An eigenspace-based approach for human fall detection using integrated time motion image and neural network. In 2008 9th International Conference on Signal Processing (pp. 1499-1503). IEEE. Beijing, China.

Frieson, C.W. (2016). Predictors of recurrent falls in community-dwelling older adults after fall-Related hip fracture. Journal of Perioperative and Critical Intensive Care Nursing, 2(2), e107.

Fu, Z., Delbruck, T., Lichtsteiner, P., \& Culurciello, E. (2008). An address-event fall detector for assisted living applications. IEEE Transactions on Biomedical Circuits and Systems, 2(2), 88-96.

Ge, C., Gu, I.Y.H., \& Yang, J. (2017, September). Human fall detection using segment-level CNN features and sparse dictionary learning. In 2017 IEEE 27th International Workshop on Machine Learning for Signal Processing (MLSP) (pp. 1-6). IEEE. Tokyo, Japan.

Harrou, F., Zerrouki, N., Sun, Y., \& Houacine, A. (2017). Vision-based fall detection system for improving safety of elderly people. IEEE Instrumentation \& Measurement Magazine, 20(6), 49-55.

Hazelhoff, L., Han, J., \& de With, P.H.N. (2008) Video-based fall detection in the home using principal component analysis. In: Blanc-Talon J., Bourennane S., Philips W., Popescu D., Scheunders P. (eds) Advanced Concepts for Intelligent Vision Systems. ACIVS 2008. Lecture Notes in Computer Science, vol 5259. Springer, Berlin, Heidelberg.

Hijaz, F., Afzal, N., Ahmad, T., \& Hasan, O. (2010, June). Survey of fall detection and daily activity monitoring techniques. In 2010 International Conference on Information and Emerging Technologies (pp. 1-6). IEEE. Bali, Indonesia.

Hsieh, Y.Z., \& Jeng, Y.L. (2017). Development of home intelligent fall detection lot system based on feedback optical flow convolutional neural network. IEEE Access, 6, 6048-6057. 
International Journal of Mathematical, Engineering and Management Sciences

Vol. 5, No. 1, 161-180, 2020

https://doi.org/10.33889/IJMEMS.2020.5.1.014

Igual, R., Medrano, C., \& Plaza, I. (2013). Challenges, issues and trends in fall detection systems. Biomedical Engineering Online, 12(1), 66. https://doi.org/10.1186/1475-925X-12-66.

Jokanovic, B., \& Amin, M. (2017). Fall detection using deep learning in range-Doppler radars. IEEE Transactions on Aerospace and Electronic Systems, 54(1), 180-189.

Jokanovic, B., Amin, M., \& Ahmad, F. (2016a, May). Radar fall motion detection using deep learning. In 2016 IEEE Radar Conference (RadarConf) (pp. 1-6). IEEE. Philadelphia, Pennsylvania, USA.

Jokanovic, B., Amin, M., Ahmad, F., \& Boashash, B. (2016 b, May). Radar fall detection using principal component analysis. In Radar Sensor Technology XX (Vol. 9829, p. 982919). International Society for Optics and Photonics. https://doi.org/10.1117/12.2225106. SPIE Defense + Security, 2016, Baltimore, Maryland, United States.

Jokanovic, B., Amin, M.G., \& Ahmad, F. (2016c, July). Effect of data representations on deep learning in fall detection. In 2016 IEEE Sensor Array and Multichannel Signal Processing Workshop (SAM) (pp. 15). IEEE. Rio de Janerio, Brazil.

Kerdegari, H., Samsudin, K., Ramli, A.R., \& Mokaram, S. (2012, June). Evaluation of fall detection classification approaches. In 2012 4th International Conference on Intelligent and Advanced Systems (ICIAS2012) (Vol. 1, pp. 131-136). IEEE. Kuala Lumpur, Malaysia.

Kim, Y., \& Ling, H. (2009). Human activity classification based on micro-Doppler signatures using a support vector machine. IEEE Transactions on Geoscience and Remote Sensing, 47(5), 1328-1337.

Klack, L., Mollering, C., Ziefle, M., \& Schmitz-Rode, T. (2010, October). Future care floor: a sensitive floor for movement monitoring and fall detection in home environments. In International Conference on Wireless Mobile Communication and Healthcare (pp. 211-218). Springer. Berlin, Heidelberg.

Kreković, M., Čerić, P., Dominko, T., Ilijaš, M., Ivančić, K., Skolan, V., \& Šarlija, J. (2012, May). A method for real-time detection of human fall from video. In 2012 Proceedings of the 35th International Convention on Information and Communication Technology, Electronics and Microelectronics (MIPRO) (pp. 1709-1712). IEEE. Opatija, Croatia.

Li, Q., Stankovic, J.A., Hanson, M.A., Barth, A.T., Lach, J., \& Zhou, G. (2009, June). Accurate, fast fall detection using gyroscopes and accelerometer-derived posture information. In 2009 Sixth International Workshop on Wearable and Implantable Body Sensor Networks (Vol. 9, pp. 138-143). IEEE. Berkeley, CA, USA.

Li, Y., Ho, K.C., \& Popescu, M. (2012). A microphone array system for automatic fall detection. IEEE Transactions on Biomedical Engineering, 59(5), 1291-1301.

Li, Y., Popescu, M., Ho, K.C., \& Nabelek, D.P. (2011, August). Improving acoustic fall recognition by adaptive signal windowing. In 2011 Annual International Conference of the IEEE Engineering in Medicine and Biology Society (pp. 7589-7592). IEEE. Boston, Massachusetts.

Li, Y., Zeng, Z., Popescu, M., \& Ho, K.C. (2010, August). Acoustic fall detection using a circular microphone array. In 2010 Annual International Conference of the IEEE Engineering in Medicine and Biology (pp. 2242-2245). IEEE. Buenos Aire.

Lie, W.N., Le, A.T., \& Lin, G.H. (2018, January). Human fall-down event detection based on 2D skeletons and deep learning approach. In 2018 International Workshop on Advanced Image Technology (IWAIT) (pp. 1-4). IEEE. Chiang Mai, Thailand.

Lim, D., Park, C., Kim, N.H., Kim, S.H., \& Yu, Y.S. (2014). Fall-detection algorithm using 3-axis acceleration: combination with simple threshold and hidden Markov model. Journal of Applied Mathematics, 2014, Article ID 896030, 8 pages. http://dx.doi.org/10.1155/2014/896030.

Liu, C.L., Lee, C.H., \& Lin, P.M. (2010). A fall detection system using k-nearest neighbor classifier. Expert Systems with Applications, 37(10), 7174-7181. 
International Journal of Mathematical, Engineering and Management Sciences

Vol. 5, No. 1, 161-180, 2020

https://doi.org/10.33889/IJMEMS.2020.5.1.014

Lu, N., Ren, X., Song, J., \& Wu, Y. (2017, August). Visual guided deep learning scheme for fall detection. In 2017 13th IEEE Conference on Automation Science and Engineering (CASE) (pp. 801-806). IEEE. Xi'an, China.

Mastorakis, G., \& Makris, D. (2014). Fall detection system using Kinect's infrared sensor. Journal of RealTime Image Processing, 9(4), 635-646.

Min, W., Cui, H., Rao, H., Li, Z., \& Yao, L. (2018). Detection of human falls on furniture using scene analysis based on deep learning and activity characteristics. IEEE Access, 6, 9324-9335.

Mohamed, O., Choi, H.J., \& Iraqi, Y. (2014, March). Fall detection systems for elderly care: a survey. In 2014 6th International Conference on New Technologies, Mobility and Security (NTMS) (pp. 1-4). IEEE. Zayed University, Dubai.

Mubashir, M., Shao, L., \& Seed, L. (2013). A survey on fall detection: principles and approaches. Neurocomputing, 100, 144-152.

Nizam, Y., Mohd, M.N.H., \& Jamil, M.M.A. (2016). A study on human fall detection systems: daily activity classification and sensing techniques. International Journal of Integrated Engineering, 8(1), 35-43.

Nunez-Marcos, A., Azkune, G., \& Arganda-Carreras, I. (2017). Vision-based fall detection with convolutional neural networks. Wireless Communications and Mobile Computing, 2017, Article ID 9474806, 16 pages. https://doi.org/10.1155/2017/9474806.

Perry, J.T., Kellog, S., Vaidya, S.M., Youn, J.H., Ali, H., \& Sharif, H. (2009, December). Survey and evaluation of real-time fall detection approaches. In 2009 6th International Symposium on High Capacity Optical Networks and Enabling Technologies (HONET) (pp. 158-164). IEEE. Alexandria, Egypt.

Popescu, M., Li, Y., Skubic, M., \& Rantz, M. (2008, August). An acoustic fall detector system that uses sound height information to reduce the false alarm rate. In 2008 30th Annual International Conference of the IEEE Engineering in Medicine and Biology Society (pp. 4628-4631). IEEE. Vancouver, BC, Canada.

Rimminen, H., Lindstrom, J., Linnavuo, M., \& Sepponen, R. (2010). Detection of falls among the elderly by a floor sensor using the electric near field. IEEE Transactions on Information Technology in Biomedicine, 14(6), 1475-1476.

Rougier, C., Meunier, J., St-Arnaud, A., \& Rousseau, J. (2011). Robust video surveillance for fall detection based on human shape deformation. IEEE Transactions on Circuits and Systems for Video Technology, 21(5), 611-622.

Shan, S., \& Yuan, T. (2010, October). A wearable pre-impact fall detector using feature selection and support vector machine. In IEEE 10th International Conference on Signal Processing Proceedings (pp. 16861689). IEEE. Singapore.

Shojaei-Hashemi, A., Nasiopoulos, P., Little, J.J., \& Pourazad, M.T. (2018, May). Video-based human fall detection in smart homes using deep learning. In 2018 IEEE International Symposium on Circuits and Systems (ISCAS) (pp. 1-5). IEEE. Firenze Fiera Spa, Florence, Italy.

Singh, M.A.F. (2014). Exercise, nutrition and managing hip fracture in older persons. Current Opinion in Clinical Nutrition \& Metabolic Care, 17(1), 12-24.

Sposaro, F., \& Tyson, G. (2009, September). IFall: An android application for fall monitoring and response. In 2009 Annual International Conference of the IEEE Engineering in Medicine and Biology Society (pp. 6119-6122). IEEE. Minneapolis, MN, USA.

Toreyin, B.U., Soyer, A.B., Onaran, I., \& Cetin, E.E. (2007). Falling person detection using multi-sensor signal processing. EURASIP Journal on Advances in Signal Processing, 2008(1), 149304. 
International Journal of Mathematical, Engineering and Management Sciences

Vol. 5, No. 1, 161-180, 2020

https://doi.org/10.33889/IJMEMS.2020.5.1.014

Tzeng, H.W., Chen, M.Y., \& Chen, J.Y. (2010, July). Design of fall detection system with floor pressure and infrared image. In 2010 International Conference on System Science and Engineering (pp. 131-135). IEEE. Amsterdam.

Uddin, M., Khaksar, W., \& Torresen, J. (2018). Ambient sensors for elderly care and independent living: A survey. Sensors, 18(7), 2027.

Verma, A., Merchant, R.A., Seetharaman, S., \& Yu, H. (2016, November). An intelligent technique for posture and fall detection using multiscale entropy analysis and fuzzy logic. In 2016 IEEE Region 10 Conference (TENCON) (pp. 2479-2482). IEEE. Singapore.

Vishwakarma, V., Mandal, C., \& Sural, S. (2007, December). Automatic detection of human fall in video. In International Conference on Pattern Recognition and Machine Intelligence (pp. 616-623). Springer. Berlin, Heidelberg.

Wang, C.C., Chiang, C.Y., Lin, P.Y., Chou, Y.C., Kuo, I.T., Huang, C.N., \& Chan, C.T. (2008, May). Development of a fall detecting system for the elderly residents. In 2008 2nd International Conference on Bioinformatics and Biomedical Engineering (pp. 1359-1362). IEEE. Shanghai, China.

Wang, J., Zhang, Z., Li, B., Lee, S., \& Sherratt, R.S. (2014). An enhanced fall detection system for elderly person monitoring using consumer home networks. IEEE Transactions on Consumer Electronics, 60(1), 23-29.

WHO (2008). World health organization: Global report on falls prevention in older age. https://www.who.int/ageing/publications/Falls_prevention7March.pdf.

Willems, J., Debard, G., Vanrumste, B., \& Goedeme, T. (2009, September). A video-based algorithm for elderly fall detection. In World Congress on Medical Physics and Biomedical Engineering (pp. 312315). Springer. Munich, Germany.

WPA, 2017, World Population Ageing, United Nations. https://www.un.org/en/development/desa/population/publications/pdf/ageing/WPA2017_Highlights.pd f.

Yang, L., Ren, Y., \& Zhang, W. (2016). 3D depth image analysis for indoor fall detection of elderly people. Digital Communications and Networks, 2(1), 24-34.

Yu, X. (2008, July). Approaches and principles of fall detection for elderly and patient. In HealthCom 200810th International Conference on e-health Networking, Applications and Services (pp. 42-47). IEEE. Singapore.

Yuwono, M., Moulton, B.D., Su, S.W., Celler, B.G., \& Nguyen, H.T. (2012). Unsupervised machine-learning method for improving the performance of ambulatory fall-detection systems. Biomedical Engineering Online, 11(1), 9.

Zerrouki, N., \& Houacine, A. (2018). Combined curve lets and hidden Markov models for human fall detection. Multimedia Tools and Applications, 77(5), 6405-6424.

Zhang T., Wang J., Xu L., Liu P. (2006) Fall Detection by Wearable Sensor and One-Class SVM Algorithm. In: Huang DS., Li K., Irwin G.W. (eds) Intelligent Computing in Signal Processing and Pattern Recognition. Lecture Notes in Control and Information Sciences, vol 345, pp. 858-863. Springer, Berlin, Heidelberg.

Zhao, F., Cao, Z., Xiao, Y., Mao, J., \& Yuan, J. (2018a). Real-time detection of fall from bed using a single depth camera. IEEE Transactions on Automation Science and Engineering, 16(3), 1018-1032.

Zhao, S., Li, W., Niu, W., Gravina, R., \& Fortino, G. (2018b). Recognition of human fall events based on single tri-axial gyroscope. In 2018 IEEE 15th International Conference on Networking, Sensing and Control (ICNSC) (pp. 1-6). IEEE. Zhuhai, China. 
International Journal of Mathematical, Engineering and Management Sciences

Vol. 5, No. 1, 161-180, 2020

https://doi.org/10.33889/IJMEMS.2020.5.1.014

Zhi, Y., \& Hui, H. (2017). A fall detection method based on two-stream convolutional neural network. Journal of Henan Normal University (Natural Science Edition), 45(3), 96-101.

Zhu, L., Zhou, P., Pan, A., Guo, J., Sun, W., Wang, L., \& Liu, Z. (2015, August). A survey of fall detection algorithm for elderly health monitoring. In 2015 IEEE Fifth International Conference on Big Data and Cloud Computing (pp. 270-274). IEEE. Dalian University of Technology, China.

Zhuang, X., Huang, J., Potamianos, G., \& Hasegawa-Johnson, M. (2009, April). Acoustic fall detection using Gaussian mixture models and GMM supervectors. In 2009 IEEE International Conference on Acoustics, Speech and Signal Processing (pp. 69-72). IEEE. Taipei, Taiwan.

Zigel, Y., Litvak, D., \& Gannot, I. (2009). A method for automatic fall detection of elderly people using floor vibrations and sound-Proof of concept on human mimicking doll falls. IEEE Transactions on Biomedical Engineering, 56(12), 2858-2867.

Original content of this work is copyright (C) International Journal of Mathematical, Engineering and Management Sciences. Uses under the Creative Commons Attribution 4.0 International (CC BY 4.0) license at https://creativecommons.org/licenses/by/4.0/ 